\title{
Additional Cytotoxic Diacetylenes from the Stony Coral Montipora sp.
}

\author{
Naseer Alam, ${ }^{a}$ Jongki Hong, ${ }^{b}$ Chong-Ok Lee,${ }^{c}$ Jae Sue Chol, ${ }^{d}$ Kwang Sik Im, ${ }^{a}$ and Jee H. Jung ${ }^{*}, a$ \\ ${ }^{a}$ College of Pharmacy, Pusan National University; Pusan 609-735, Korea: ${ }^{b}$ Korea Basic Science Institute; Seoul, Korea: \\ ${ }^{c}$ Korea Research Institute of Chemical Technology; Taejon, Korea: and ${ }^{d}$ Pukyung National University; Pusan, Korea. \\ Received November 12, 2001; accepted January 22, 2002
}

\begin{abstract}
Three new diacetylenes $(1,4,6)$ have been isolated as cytotoxic constituents from the methanolic extract of the stony coral Montipora sp. The structures have been elucidated on the basis of spectroscopic evidence. The compounds were evaluated for cytotoxicity against a small panel of human tumor cell lines and showed moderate to significant activity.
\end{abstract}

Key words stony coral; Montipora sp.; diacetylene; cytotoxicity

The secondary metabolites of the stony corals so far investigated are few but diverse. ${ }^{1)}$ Among them, diacetylene derivatives have been isolated from the genera Montipora and Pectinia. ${ }^{1-4)}$ Montipora sp. (Scleractinia, Coelenterata) is a hermaphroditic coral and has been investigated from both ecological and chemical viewpoints.

We previously reported several new diacetylenic compounds (montiporyne A-M) from Montipora sp. collected from Korean waters. ${ }^{1,2)}$ Some have shown significant cytotoxic activity. Subsequently, we isolated additional congeners by activity-guided fractionation of the methanolic extract of the same coral. Here, we report the isolation and structure elucidation of three additional diacetylenes $(\mathbf{1}, \mathbf{4}, \mathbf{6})$ that share the same gross structure with the compounds reported earlier. ${ }^{1)}$

Compound $\mathbf{1}$ was isolated as a light yellow oil. ${ }^{1} \mathrm{H}-\mathrm{NMR}$ signals of 1 resembled those of methyl montiporate B (2) reported earlier from the same source. ${ }^{1)}$ Two singlets of two protons each at $\delta 4.33$ and 4.17 were due to $\mathrm{H}-1$ and $\mathrm{H}-2^{\prime}$ protons, respectively, while the methoxy protons resonated at $\delta$ 3.74. The signals at $\delta 5.80$ (ddt), 5.00 (dd), and 4.95 (dd) were assigned to the terminal olefinic protons. A triplet at $\delta$ 2.30 was attributed to the $\alpha$-acetylenic methylene protons (H-6). The compound showed an $[\mathrm{M}+\mathrm{Na}]^{+}$ion peak at $\mathrm{m} / \mathrm{z}$ 271 in the FAB-MS analysis that matched well with the molecular formula $\mathrm{C}_{15} \mathrm{H}_{20} \mathrm{O}_{3}$ which was assumed from the NMR data. All this evidence suggests that $\mathbf{1}$ is a lower-mass analogue of methyl montiporate $\mathrm{B}\left(2, \mathrm{C}_{17} \mathrm{H}_{24} \mathrm{O}_{3}\right)$ and the structure was determined to be methyl 2-O-(11-dodecene-2,4diynyl)-2-hydroxy ethanoate.

Compound 4 showed an isolated oxygenated methylene ( $\delta$ $4.24, \mathrm{H}-1)$ and an $\alpha$-acetylenic methylene ( $\delta 2.29, \mathrm{H}-6)$ characteristic of montiporynes. Two triplets at $\delta 3.59(J=5.5 \mathrm{~Hz})$
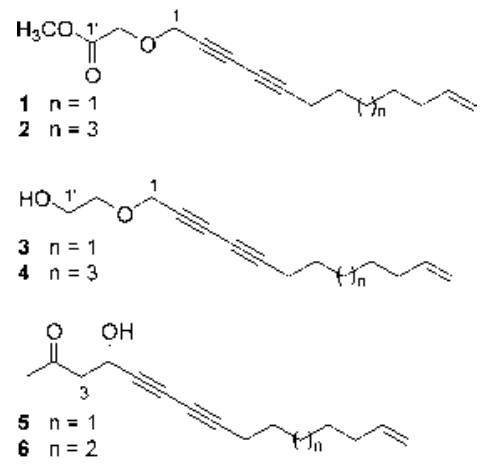

and $3.66(J=5.5 \mathrm{~Hz})$ were similar to those for the $\mathrm{H}-2^{\prime}$ and $\mathrm{H}-1^{\prime}$ protons in montiporyne $\mathrm{H}(3)$. The FAB-MS showed the $[\mathrm{M}+\mathrm{Na}]^{+}$ion at $m / z 271$, and thus the molecular formula was deduced to be $\mathrm{C}_{16} \mathrm{H}_{24} \mathrm{O}_{2}$, which showed that compound 4 was a higher-mass analogue of montiporyne $\mathrm{H}\left(3, \mathrm{C}_{14} \mathrm{H}_{20} \mathrm{O}_{2}\right) .{ }^{1}$ Thus the structure was assigned as 2-O-(13-tetradecene-2,4diynyl)-1,2-ethanediol.

Compound 6 was a $\beta$-hydroxy ketone derivative similar to montiporyne $\mathrm{J}\left(\mathbf{5}, \mathrm{C}_{15} \mathrm{H}_{20} \mathrm{O}_{2}\right){ }^{1}{ }^{1)}$ Two doublets of doublets at $\delta$ $2.78(J=16.5,5.5 \mathrm{~Hz})$ and $2.86(J=16.5,8.0 \mathrm{~Hz})$ were due to $\mathrm{H}-3$ protons, while the $\mathrm{H}-4$ proton resonated as a doublet of doublets at $\delta 4.78(J=8.0,5.5 \mathrm{~Hz})$. Signals corresponding to an $\alpha$-acetylenic methylene and a monosubstituted olefin were also observed. The FAB-MS showed an $[\mathrm{M}+\mathrm{Na}]^{+}$ion at $m / z 269$, which corresponded to the molecular formula $\mathrm{C}_{16} \mathrm{H}_{22} \mathrm{O}_{2} \mathrm{Na}$. Thus the structure was elucidated to be 4-hydroxy-15-hexadecene-5,7-diyne-2-one. The stereochemistry at C-4 remains to be determined.

The isolated compounds were tested against a small panel of human cancer cell lines and the results are shown in Table 1. The compounds showed an structure-activity relationship (SAR) profile similar to their earlier reported congeners. ${ }^{1)}$ Methyl montiporate C (1) was active only against a skin cancer cell line. Compound $\mathbf{4}$ was moderately active, while $\mathbf{6}$, with $\beta$-hydroxy ketone functionality, exhibited more potent cytotoxicity.

\section{Experimental}

General NMR spectra were recorded on a Varian INOVA 500 spectrometer. Chemical shifts were reported in reference to the respective residual solvent peaks ( $\delta_{\mathrm{H}} 3.3$ and $\delta_{\mathrm{C}} 49.0$ for $\left.\mathrm{CD}_{3} \mathrm{OD}\right)$. LR-FAB-MS data were obtained using a JEOL JMS-HX110/110A. HPLC was performed on a Gilson 370 pump with a YMC ODS-H80 $(250 \times 10 \mathrm{~mm}$ i.d., S- $4 \mu \mathrm{m}, 80 \AA)$ column using a Shodex RI-71 detector.

Animal Material The animals were collected by hand using scuba gear at a depth of $8 \mathrm{~m}$ in November 1996, along the shore of Mundo, Cheju Is-

Table 1. Cytotoxicities $\left(\mathrm{ED}_{50}, \mu \mathrm{g} / \mathrm{ml}\right)$ of Compounds $\mathbf{1}, \mathbf{4}$, and $\mathbf{6}$ against Human Solid Tumor Cells ${ }^{a)}$

\begin{tabular}{lrrcrc}
\hline \hline Compound & A549 & SK-OV-3 & SK-MEL-2 & XF498 & HCT15 \\
\hline $\mathbf{1}$ & $>30$ & 27.1 & 5.51 & $>30$ & $>30$ \\
$\mathbf{4}$ & 11.29 & 13.80 & 4.36 & 12.97 & 8.43 \\
$\mathbf{6}$ & 6.20 & 4.78 & 3.85 & 7.24 & 6.94 \\
Doxorubicin & 0.02 & 0.13 & 0.03 & 0.08 & 0.04 \\
Cisplatin & 0.75 & 1.09 & 2.18 & 1.18 & 0.85 \\
\hline
\end{tabular}

a) A549, human lung cancer; SK-OV-3, human ovarian cancer; SK-MEL-2, human skin cancer; XF498, human CNS cancer; HCT15, human colon cancer. 
land, Korea, and were described in a previous report. ${ }^{2)}$ A voucher specimen was deposited in the Natural History Museum, Ewha Womans University (voucher no. EWUA. Ant. 961104).

Extraction and Isolation The frozen coral $(2.5 \mathrm{~kg}$, wet wt.) was extracted with $\mathrm{MeOH}$ at room temperature. Guided by the brine shrimp lethality assay, ${ }^{5)}$ the $\mathrm{MeOH}$ extract was partitioned between $\mathrm{H}_{2} \mathrm{O}$ and EtOAc. The EtOAc layer was further partitioned between $\mathrm{H}_{2} \mathrm{O}$ and $\mathrm{CHCl}_{3}$ to afford $8.8 \mathrm{~g}$ of the $\mathrm{CHCl}_{3}$ layer $\left(\mathrm{LD}_{50} 30-86 \mu \mathrm{g} / \mathrm{ml}\right)$ which was subjected to a reversephase medium pressure liquid chromatography (MPLC) (YMC gel ODS-A, $60 \AA 500 / 400$ mesh) eluted with a step gradient solvent system of $25 \rightarrow 0 \%$ $\mathrm{H}_{2} \mathrm{O} / \mathrm{MeOH}$ to obtain 14 fractions $(1-14)$. Fraction $3(3.1 \mathrm{~g})$ was very active in the brine shrimp test and was further separated into 26 fractions on a reverse-phase MPLC (YMC gel ODS-A, $60 \AA, 500 / 400$ mesh), eluted with a step gradient solvent system of $20 \rightarrow 0 \% \mathrm{H}_{2} \mathrm{O} / \mathrm{MeOH}$. Sub fraction 3-18 was repeatedly chromatographed on HPLC (YMC ODS-H80, $250 \times 10 \mathrm{~mm}$ i.d., S-4 $\mu \mathrm{m}, 80 \AA$ ) eluted with $90 \% \mathrm{MeOH} / \mathrm{H}_{2} \mathrm{O}$ to yield compound 1 (1 mg). Compound $4(0.6 \mathrm{mg})$ was purified from subfraction 3-21 using $90 \%$ $\mathrm{MeOH} / \mathrm{H}_{2} \mathrm{O}$ on the same column. Compound $6(0.8 \mathrm{mg})$ was purified from sub fraction 3-11 using the same column with $80 \% \mathrm{MeOH} / \mathrm{H}_{2} \mathrm{O}$ as the mobile phase.

Methyl Montiporate C (1): Light yellow oil. UV $(\mathrm{MeOH}) \lambda_{\max } \mathrm{nm}(\log \varepsilon)$ : 286 (3.40), 226 (3.76). IR (film) $v_{\max } \mathrm{cm}^{-1}: 3376,2927,2856,1716,1071$. ${ }^{1} \mathrm{H}-\mathrm{NMR}\left(\mathrm{CD}_{3} \mathrm{OD}\right) \quad \delta: 1.25-1.55(6 \mathrm{H}, \mathrm{m}, \mathrm{H}-7-\mathrm{H}-9), 2.07$ (quart, $J=7.5 \mathrm{~Hz}, \mathrm{H}-10), 2.30$ (t, $J=7.0 \mathrm{~Hz}, \mathrm{H}-6), 3.74\left(\mathrm{~s}, \mathrm{OCH}_{3}\right), 4.17$ (s, H-2'), 4.33 (s, H-1), 4.95 (dd, $J=10.0,2.5 \mathrm{~Hz}, \mathrm{H}-12), 5.00$ (dd, $J=17.0,2.5 \mathrm{~Hz}, \mathrm{H}-$ 12), 5.80 (ddt, $J=17.0,10.0,7.0 \mathrm{~Hz}, \mathrm{H}-11) .{ }^{13} \mathrm{C}-\mathrm{NMR}\left(\mathrm{CD}_{3} \mathrm{OD}\right) \delta: 172.0$ $\left(\mathrm{C}-1^{\prime}\right), 139.8(\mathrm{C}-11), 115.4(\mathrm{C}-12), 82.4,72.7,71.9,65.2(\mathrm{C}-2-5), 67.2(\mathrm{C}-$ $\left.2^{\prime}\right), 59.8(\mathrm{C}-1), 52.3\left(\mathrm{OCH}_{3}\right), 34.4(\mathrm{C}-10), 29.3-29.8$ (C-7-9), 19.7 (C-6). FAB-MS $m / z: 271[\mathrm{M}+\mathrm{Na}]^{+}$.

Dihomomontiporyne $\mathrm{H}(4)$ : Light yellow oil. ${ }^{1} \mathrm{H}-\mathrm{NMR}\left(\mathrm{CD}_{3} \mathrm{OD}\right) \delta: 1.25-$ $1.55(10 \mathrm{H}, \mathrm{m}, \mathrm{H}-7-\mathrm{H}-11), 2.05$ (quart, $J=7.0 \mathrm{~Hz}, \mathrm{H}-12), 2.29(\mathrm{t}, J=7.0 \mathrm{~Hz}$, H-6), 3.59 (t, $\left.J=5.5 \mathrm{~Hz}, \mathrm{H}-2^{\prime}\right), 3.66$ (t, $\left.J=5.5 \mathrm{~Hz}, \mathrm{H}-1^{\prime}\right), 4.24$ (s, H-1), 4.94 (dd, $J=10.0,2.0 \mathrm{~Hz}, \mathrm{H}-14$ ), 4.99 (dd, $J=17.2,2.0 \mathrm{~Hz}, \mathrm{H}-14$ ), 5.80 (ddt, $J=17.2,10.0,6.5 \mathrm{~Hz}, \mathrm{H}-13) .{ }^{13} \mathrm{C}-\mathrm{NMR}\left(\mathrm{CD}_{3} \mathrm{OD}\right) \delta: 139.8(\mathrm{C}-13), 114.9(\mathrm{C}-$ 14), 81.9, 72.8, 72.0, 65.3 (C-2-5), $72.4\left(\mathrm{C}-2^{\prime}\right), 61.9\left(\mathrm{C}-1^{\prime}\right), 59.7$ (C-1), 34.6 (C-12), 29.2-29.6 (C-7-11), 19.7 (C-6). FABMS $m / z: 271$ $[\mathrm{M}+\mathrm{Na}]^{+}$

Homomontiporyne $\mathrm{J}(6)$ : Light yellow oil. ${ }^{1} \mathrm{H}-\mathrm{NMR}\left(\mathrm{CD}_{3} \mathrm{OD}\right) \delta: 1.28$ $1.52(8 \mathrm{H}, \mathrm{m}, \mathrm{H}-10-\mathrm{H}-13), 2.06$ (quart, $J=7.0 \mathrm{~Hz}, \mathrm{H}-14), 2.16$ (s, H-1), 2.28 (t, $J=7.0 \mathrm{~Hz}, \mathrm{H}-9), 2.78$ (dd, $J=16.5,5.5 \mathrm{~Hz}, \mathrm{H}-3$ ), 2.86 (dd, $J=16.5$, $8.0 \mathrm{~Hz}, \mathrm{H}-3), 4.78$ (dd, $J=8.0,5.5 \mathrm{~Hz}, \mathrm{H}-4), 4.94$ (dd, $J=10.2,2.0 \mathrm{~Hz}, \mathrm{H}-$ 16), 4.99 (dd, $J=17.0,2.0 \mathrm{~Hz}, \mathrm{H}-16$ ), 5.80 (ddt, $J=17.0,10.2,6.8 \mathrm{~Hz}, \mathrm{H}-15$ ). ${ }^{13} \mathrm{C}$-NMR $\left(\mathrm{CD}_{3} \mathrm{OD}\right) \delta$ : $207.6(\mathrm{C}-2), 139.9(\mathrm{C}-15), 115.0(\mathrm{C}-16), 82.3,76.8$, 70.1, 65.3 (C-5-8), 59.0 (C-4), 51.8 (C-3), 34.6 (C-14), 29.3-29.8 (C10-13), 30.6 (C-1), 19.7 (C-9). FAB-MS $m / z: 269[\mathrm{M}+\mathrm{Na}]^{+}$.

Acknowledgments Thanks are due to Professor Jun-Im Song for the identification of the coral. A grant from Pusan National University is gratefully acknowledged.

\section{References and Notes}

1) Alam N., Bae B. H., Hong J.-K., Lee C.-O., Im K. S., Jung J. H., J. Nat. Prod., 64, 1059-1063 (2001) and references cited therein

2) Bae B. H., Im K. S., Choi W. C., Hong J.-K., Lee C.-O., Choi J. S., Son B. W., Song J.-I., Jung J. H., J. Nat. Prod., 63, 1511-1514 (2000).

3) Coll J. C., Bowden B. F., Meehan G. V., Konig G. M., Carroll A. R., Tapiolas D. M., Alino P. M., Heaton A., De Nys R., Leone P. A., Maida M., Aceret T. L., Willis R. H., Babcock R. C., Willis B. L., Florian Z., Clayton M. N., Miller R. L., Mar. Biol., 118, 177-182 (1994).

4) Higa T., Tanaka J., Kohagura T., Wauke T., Chem. Lett., 1990, 145148 (1990).

5) Meyer B. N., Ferrigni N. R., Putnam J. E., Jacobsen L. B., Nichols D. E., McLaughlin J. L., Planta Medica, 45, 31-34 (1982). 